\title{
Impact of Nanotechnology on the Future Agriculture: The Road toward Smart Agriculture (Abstract)
}

\author{
Taher A. Salah \\ Nanotechnology Research Centre, British University in Egypt \\ Corresponding author: $\underline{\mathrm{t} 1 \mathrm{sal} a \mathrm{~h} @ \text { hotmail.com }}$
}

\begin{abstract}
Over the past few years, scientific data indicate that nanotechnology has potential impact on the agrifood sector, minimizing adverse problems of agricultural practices on environment and human health, improving food security and productivity (as required by the predicted rise in global population), while promoting social and economic equity.

Smart agriculture (SA) is an approach that helps to guide actions needed to transform and reorient agricultural systems to effectively support development and ensure food security according to a changing climate. Nanotechnology draw the road toward the smart agriculture and aims to tackle three main objectives of SA: sustainably increasing agricultural productivity and incomes; adapting and building resilience to climate change; and reducing and/or removing greenhouse gas emissions, where possible. Approaches of Nanotechnology provide the means to help stakeholders from local to national and international levels identify agricultural strategies suitable to their local conditions.

In this context, we select and report on recent trends in nanomaterial-based systems and nanodevices that could provide benefits on the food supply chain specifically on sustainable intensification, and management of soil and waste. Among others, nanomaterials for controlled-release of nutrients, pesticides and fertilizers in crops are described as well as nanosensors for agricultural practices, food quality and safety.
\end{abstract}

Key words: Nanotechnology, Agrifood, Smart Agriculture, Nanomaterials, Water. 
